\title{
Politique
}

\section{Fondements de la politique française en matière d'informatisation}

\section{Sylvain Vézina}

Numéro 22, automne 1992

URI : https://id.erudit.org/iderudit/040730ar

DOI : https://doi.org/10.7202/040730ar

Aller au sommaire du numéro

Éditeur(s)

Société québécoise de science politique

ISSN

0711-608X (imprimé)

1918-6584 (numérique)

Découvrir la revue

Citer cet article

Vézina, S. (1992). Fondements de la politique française en matière

d'informatisation. Politique, (22), 75-99. https://doi.org/10.7202/040730ar
Résumé de l'article

Comprendre l'état de développement actuel des politiques sectorielles en matière d'informatisation de l'administration publique suppose un retour aux projets de société à la base de leur élaboration et application. L'étude critique de deux rapports officiels (S. Nora/A. Mine, P. Lemoine), laquelle porte sur la conception du pouvoir et du changement véhiculée dans le traitement de leurs problématiques respectives, dévoile un écart important au plan de

l'orientation paradigmatique et conséquemment de la stratégie à adopter pour gérer la mutation technologique. Cette analyse tend à démontrer que les plus lourdes conséquences de l'informatisation de la société ne procèdent pas de l'introduction de l'outil en lui-même mais des projets de société formulés à son endroit et de la conception de la réalité qui les sous-tend. 


\title{
FONDEMENTS DE LA POLITIQUE FRANÇAISE EN MATIÈRE D'INFORMATISATION
}

\author{
Sylvain Vézina \\ Universite de Moncton
}

Comprendre l'état de développement actuel des politiques sectorielles en matière d'informatisation de l'administration publique suppose un retour aux projets de société à la base de leur élaboration et application. L'étude critique de deux rapports officiels (S. Nora/A. Minc., P. Lemoine), laquelle porte sur la conception du pouvoir et du changement véhiculée dans le traitement de leurs problématiques respectives, dévoile un écart important au plan de l'orientation paradigmatique et conséquemment de la stratégie à adopter pour gérer la mutation technologique. Cette analyse tend à démontrer que les plus lourdes conséquences de l'informatisation de la société ne procèdent pas de l'introduction de l'outil en lui même mais des projets de société formulés à son endroit et de la conception de la réalité qui les sous-tend.

Les politiques sectorielles d'informatisation de l'administration publique posent souvent des problèmes. Inadéquates ou mal comprises, elles sont l'objet de critiques, de résistances ou, carrément, de sabotage. Plusieurs facteurs pourraient expliquer ce phénomène. L'un d'eux se rapporte aux origines du processus. Il est alors question d'évaluer les grandes orientations nationales, fondements des politiques en matière de nouvelles technologies, lesquelles guideront le développement ultérieur des politiques sectorielles. Nous procéderons donc, dans ce texte, à l'analyse de deux documents officiels, le rapport Nora/Minc ${ }^{1}$ et le rapport Lemoine ${ }^{2}$, dont la pertinence est d'établir

1 S. Nora et A. Minc, L'informatisation de la société, Paris, La Documentation française, 1978.

2 P. Lemoine, Les technologies d'information, enjeu stratégique pour la modernisation économique et sociale, Paris, La Documentation Française, 1983.

Revue québécoise de science politique, no 22, automne 1992. 
les bases des politiques adoptées en France en matière d'exploitation des développements informatiques récents.

Nous postulons la représentativité de ces deux rapports, car ils nous paraissent former une sorte de cristallisation du discours et des aspirations autour desquels graviteront les différentes actions sectorielles liées à la modernisation de la société française. Ils reflètent assez fidèlement les craintes et les espoirs au sujet de l'incidence de l'informatisation de la société tant au plan politique, économique que social.

Les deux rapports étudiés ayant déjà fait l'objet de discussions plus ou moins approfondies ${ }^{3}$, nous avons choisi d'orienter plus particulièrement notre analyse sur leurs fondements conceptuels. Chacun de ces rapports reflète une conception de la réalité, transmise par les auteurs. Leur mode d'appréhension du phénomène informatique influencera non seulement le diagnostic qu'ils posent, mais également l'orientation des mesures qu'ils prescrivent. Par exemple, Lucien Sfez ${ }^{4}$, dans un ouvrage passionnant, suggère de saisir l'approche des théoriciens qui ont pensé notre rapport à la technique et à la communication selon trois visions du monde, traduites dans des métaphores qu'il dit actives et polyvalentes : celles de la machine, de l'organisme et de Frankenstein.

Notre étude des fondements des rapports Nora/Minc et Lemoine porte principalement sur deux concepts fondamentaux en science politique : pouvoir et changement. Si nous avons choisi d'articuler notre analyse à partir de ces deux concepts, c'est qu'ils nous paraissent se retrouver au centre de toute réflexion sur l'informatisation de nos sociétés.

Quelle que soit l'approche adoptée, l'informatique sera invariablement associée au changement. Après l'agriculture et l'industrialisation, l'informatisation de la société caractériserait "la troisième vague" postindustrielle ${ }^{5}$. Que l'on soit optimiste, pessimiste ou même sceptique, l'informatique fascine en ce qu'elle

3 Surtout le rapport Nora/Minc pour lequel on peut citer entre autres : J. Bounine, B. Lussato, Télématique... ou Privatique? Questions à Simon Nora et Alain Minc, Boulogne, Éditions d'informatique, 1980; A. Vitalis, Informatique, pouvoir et liberté, Paris, Economica, 1981.

$4 \quad$ L. Sfez, Critique de la communication, Paris, Seuil, 1988.

5 D. Bell, The Coming of Post-Industrial Society, New York, Basic Books, 1973; A. Toffler, La troisieme vague, Paris, Denoël, 1981. 
se présente comme un outil puissant, susceptible de favoriser la transformation d'un ordre social fondé de plus en plus nettement sur le dogme de la rationalité. De la même manière, les nouvelles technologies sont très souvent étudiées en relation avec le phénomène du pouvoir. Le lien communément établi entre savoir et pouvoir nous oblige à penser le rôle que tient l'informatique dans nos rapports sociaux.

Ainsi, après avoir fait ressortir clairement la problématique formulée dans chacun des deux rapports, nous tenterons d'identifier les conceptions transmises par les auteurs à propos des phénomènes du changement et du pouvoir. De cette manière, nous pourrons mieux saisir le sens profond de leurs analyses et, par corollaire, déceler les implications de ces rapports au niveau même des politiques sectorielles. Nous espérons contribuer à la réflexion sur la pertinence des développements actuels en matière d'informatisation du secteur public non seulement en France, mais dans l'ensemble de nos sociétés modernes.

Toutefois, un tel exercice nécessite une réflexion préalable sur le sens que l'on prête traditionnellement aux concepts de pouvoir et de changement. Sans être exhaustive, la discussion suivante est susceptible de révéler l'ambiguïté qui entoure I'utilisation de ces notions ${ }^{6}$.

\section{La notion de pouvoir}

II semble y avoir autant de définitions du pouvoir qu'il y a de théories ou même de méthodes d'analyse. G. Bergeron ${ }^{7}$ estime que ce concept ne peut faire l'objet d'un usage scientifique étant donné son caractère vague et ambigu, issu de préjugés et de passions idéologiques.

Signalons tout d'abord la tendance fréquente à confondre pouvoir et gouvernement. Elle semble procéder d'une crainte farouche du pouvoir, perçu comme source de désordre. Le pouvoir

- Pour une analyse plus fine de ces notions, voir notamment l'analyse présentée par G. Morgan à propos de la théorie des organisations. G. Morgan, Images of Organization, Newbury Park, London, New Delhi, Sage Publications, 1989.

7 G. Bergeron, Le fonctionnement de l'État, Paris, A. Colin, 1965. 
doit ici assurer la cohésion de l'ensemble de la société ${ }^{8}$. Comme I'enseigne Rousseau, le gouvernement est un être collectif, infiniment plus important que les individus, détenteur du pouvoir souverain de définir la volonté générale dans le respect d'un intérêt commun qui transcende les intérêts particuliers égoïstes. La finalité d'une telle entreprise serait de supprimer le pouvoir avec, pour conséquence inévitable, la perte des libertés individuelles et la réduction du citoyen à l'état de machine.

Le défaut d'une telle perception est de considérer le pouvoir sous une forme limitée et institutionnelle. Or, la majorité des auteurs, considérant qu'il pénètre toute collectivité humaine selon des formes et des modalités diverses, s'entendent maintenant pour affirmer son caractère diffus.

Il ne s'agit pas de nier l'importance des institutions dans l'aménagement des relations de pouvoir. Mais de suggérer qu'il faut plutót analyser les institutions a partir des relations de pouvoir et non l'inverse; et que le point d'ancrage fondamental de celles-ci, même si elles prennent corps et se cristallisent dans une institution, est à chercher en deçàे.

Dorénavant, le pouvoir ne se limite plus à l'autorité, à l'État ou à l'ordre établi, mais doit être envisagé comme le fait de l'action sociale en soi.

Le pouvoir est-il un attribut systémique ou relationnel ? La vision systémique, fondée généralement sur une conception fonctionnaliste du pouvoir, fait référence à la capacité d'un système à atteindre ses buts ${ }^{10}$. Selon cette vision, le pouvoir représente une notion interchangeable, $c^{\prime}$ est-à-dire transposable d'une relation à l'autre. Cette lecture évince toute idée de conflit entre les individus et les groupes en présupposant le consensus

8 B. de Jouvenel, Du pouvoir, Paris, Hachette, 1972.

- M. Foucault, "Deux essais sur le sujet du pouvoir», in $\mathrm{H}$. Dreyfus et P. Rabinow, Michel Foucault, Un parcours philosophique, Paris, Gallimard, 1984, p. 316.

10 T. Parson, Politics and Social Structure, New York, Free Press, 1969. 
autour de buts communs. On y trouve des systèmes sociaux parfaitement réglés où les acteurs sont réduits à des fonctions abstraites.

À l'opposé, la vision relationnelle du pouvoir favorise les interactions entre individus et groupes. Ainsi, M. Crozier et E. Friedberg $^{11}$, en développant une problématique fondée sur le concept de jeu, réfutent l'idée d'une détention personnelle du pouvoir, arguant que le pouvoir, au lieu d'être un attribut, naît plutôt de la relation. M. Foucault, dans le même sens, affirme que le pouvoir, loin d'être un objet acquis et arraché au gré des événements, constitue plutôt un exercice, un mode d'action de certains en relation avec les autres. On ne peut donc parler, selon lui, de concentration ou de distribution du pouvoir car celui-ci n'existe qu'en acte.

\section{La notion de changement}

La notion de changement social ne connaît pas tout à fait les difficultés sémiologiques relevées à propos du pouvoir. Cela ne signifie aucunement que l'on puisse le saisir simplement, c'est-àdire sans s'interroger au préalable sur son sens et avant tout sur son expression. Puisque le changement en soi semble constituer un phénomène courant et incontesté, la question fondamentale qui se pose à son sujet concerne la dynamique même de ses manifestations. C'est à ce niveau que se présentent les difficultés, car le changement social apparaît comme un phénomène complexe dont l'expression prend des formes multiples et variées. S'agit-il d'un phénomène naturel dont l'étude permet de dégager des lois, ou s'agit-il d'un "artefact" résultant d'un processus de création collective? Les théories relatives au changement social se distinguent-elles de l'orientation prise par les historiens ?

Pour les philosophes du $\mathrm{XIX}^{\circ}$ siècle, le changement social pouvait être saisi, à l'instar des phénomènes physiques ou mathématiques, à travers la découverte des lois régissant la dynamique de toutes les sociétés. II s'agissait donc de réduire le changement à une formule unique, valant tant sur le plan de l'espace que sur celui du temps. Cette orientation positiviste devait permettre l'émergence des théories évolutionnistes.

11 M. Crozier et E. Friedberg, L'acteur et le système, Paris, Seuil, 1977. 
Parmi les tenants de cette vision, nous retiendrons les noms de H. Spencer ${ }^{12}$, qui appliqua les lois de la mécanique à l'évolution de la vie sociale dans toutes ses formes, et de L.H. Morgan $^{13}$ qui voit un lien entre évolution sociale et facteurs technologiques. Ce dernier devait d'ailleurs influencer, par ses travaux, la théorie marxiste du politique, qui voit dans la lutte des classes et l'innovation technologique les facteurs dominants de l'évolution sociale ${ }^{14}$.

Face à la diversité des changements sociaux, cette idée d'un évolutionnisme linéaire n'a pas pu résister à la critique. Les ethnologues, notamment $F$. Boas ${ }^{15}$, se sont alors intéressés au processus de diffusion des traits culturels, en écartant la tendance, dangereuse, à établir des lois de la transformation. Cette nouvelle orientation devait permettre de concevoir le changement davantage comme un processus. On verra notamment apparaître le concept $d^{\prime}$ innovation défini par l'économiste J.A. Schumpeter ${ }^{16}$ comme $^{\circ}$ "l'exécution de combinaisons nouvelles", définition qui fait appel à l'idée d'invention.

Pour R. Boudon, le changement social ne résulte pas exclusivement des conflits entre des intérêts contradictoires; il découle également des effets pervers non recherchés par les acteurs, et que certains ou l'ensemble d'entre eux jugent indésirables. Or, ces effets inattendus, fréquents, passeraient souvent inaperçus. L'auteur se déclare, par ailleurs, en faveur de "l'individualisme méthodologique ${ }^{17}$ " qu'il considère comme le seul moyen de réaliser une analyse du changement social même macroscopique.

Dans une même optique, M. Crozier et E. Friedberg considèrent que le changement n'obéit pas à des lois historiques

\footnotetext{
12 H. Spencer, Principles of Sociology, London, Macmillan, 1969.

13 L. H. Morgan, La société archaïque, Paris, Anthropos, 1971.

14 On retrouve cette lecture notamment dans la notion d'historicité développée par Hegel, Leçons sur la philosophie de I'histoire, Paris, Gallimard, 1967.

15 F. Boas, Anthropology and Modern Life, New York, Norton, 1962.

16

J. A. Schumpeter, Théorie de l'évolution économique, Paris, Dalloz,
} 1935.

17 Voir R. Bourdon, La place du désordre, Paris, PUF, 1984. 
et ne résulte pas de l'imposition inévitable d'un modèle rationnel d'organisation sociale. Le changement reposerait plutôt sur la volonté des acteurs qui, en changeant dans leurs relations réciproques, transforment l'organisation sociale. Le changement représente donc ici un construit. Contrairement aux systémistes $^{18}$, qui s'intéressent plus à la régulation et à l'équilibre du système, l'analyse stratégique postule que le changement est tout aussi nécessaire à l'organisation que la recherche de sa stabilité.

Analyser séparément la perception du pouvoir et celle du changement a pour principal défaut de négliger le lien que l'on peut établir entre ces deux concepts. En effet, ils apparaissent comme inextricablement liés. Étudier le pouvoir des individus et des groupes peut se résumer à l'analyse de leur capacité à maîtriser le changement soit en termes positifs, par sa promotion, soit en termes négatifs, en y résistant.

Selon certains, pouvoir et changement présumeraient comme condition préalable commune la liberté des individus, c'est-à-dire leur capacité relative à influencer l'ordre des choses. Ainsi, de la même manière que le changement $s$ 'inscrit dans des relations de pouvoir qui en constituent la source, il implique potentiellement une transformation de la configuration du pouvoir. Crozier et Friedberg $^{19}$ iront jusqu'à dire que les relations de pouvoir constituent un obstacle au changement en même temps que leur transformation représente l'essence même du changement. Bref, il semble indispensable pour comprendre l'un de faire référence à l'autre. C'est pourquoi nous conclurons notre analyse des deux rapports précités par une mise en relation de la perception qu'ont leurs auteurs de ces deux concepts et de leurs conséquences sur l'orientation des actions préconisées.

\section{Les problématiques}

\subsection{L'informatisation de la société (Rapport Nora/Minc)}

Lorsque le 28 janvier 1976, le Président Valéry Giscard d'Estaing confie à Simon Nora la direction d'une commission d'étude chargée de réfléchir sur les orientations à imprimer à 1951.

18 Voir notamment T. Parson, The Social System, Glencoe (III.), Free Press,

18 M. Crozier et E. Friedberg, L'acteur et le système, Paris, Seuil, 1977. 
l'informatisation de la société française, la préoccupation principale du gouvernement est la recherche de moyens de lutte contre la crise économique qui, du reste, affecte le monde entier.

Dans ce contexte, personne ne sera surpris d'apprendre que les cosignataires de ce rapport sont tous deux issus de l'Inspection générale des finances, $S$. Nora y étant inspecteur général et $A$. Minc inspecteur adjoint. On estime généralement qu'il s'agit de personnalités penchant plutôt à̀ gauche.

Le rapport Tricot, qui portait sur la protection des libertés ${ }^{20}$, examinait la portée juridique de la question informatique; le rapport Nora/Minc, pour sa part, se concentre sur sa dimension économique. Concrètement, la problématique fondamentale qui motive les auteurs de ce rapport se révèle sous ses multiples aspects dans l'énoncé suivant : "réconcilier contraintes externes, emploi et consensus social" (Nora/Minc, p. 33). II ne s'agit donc pas uniquement d'une réflexion concernant l'incidence des nouveaux développements informatiques sur la société française qui nous est proposée, mais, plus largement, d'un projet de société devant permettre de faire face aux nouveaux défis induits par l'avènement de ce qu'il convient d'appeler une société informatisée. Défis qui, répétons-le, tournent principalement autour de préoccupations économiques (Nora/Minc, p. 10).

Les préoccupations des auteurs concernant l'équilibre économique sont motivées par un triple malaise subi par la France : déficit de la balance commerciale, affaiblissement de la demande interne et accroissement constant du chômage. Or, l'équilibre recherché consiste à promouvoir la modernisation de l'économie française par l'informatisation, considérée comme une source de productivité, sans pour autant compromettre la situation de I'emploi, menacée pourtant par ce même outil (Nora/Minc, p. 23).

Malgré l'omniprésence de cette triple problématique, on remarque, tout au long de ce rapport, que la question qui préoccupe avant tout les commissaires est celle de la souveraineté nationale. Cette préoccupation touche aussi bien l'élaboration de la politique informatique que l'implantation et la fabrication du matériel.

II n'est possible d'opérer cette mutation des structures économiques et sociales

20 B. Tricot, Rapport de la commission informatique et liberté, Paris, La Documentation française, 1975. 
que si la France échappe aux pressions excessives d'États ou de groupes étrangers, dont les objectifs peuvent contrarier les siens. Son succès peut renforcer l'indépendance nationale. Mais sa mise en cuvre suppose que celle-ci ne soit pas compromise au départ (Nora/Minc, p. 10).

Dès lors, ce n'est plus l'informatique en soi qui semble devoir être considérée, mais la télématique issue de la conjonction entre l'ordinateur et les télécommunications. En permettant le décloisonnement des multiples banques d'information, la télématique assure l'établissement de réseaux à travers lesquels l'information franchit non seulement les frontières des organisations, mais aussi celles des nations.

Or, comment la France parviendra-t-elle à maintenir son indépendance devant la position dominante des États-Unis en cette matière ? Comment éviter qu'une compagnie multinationale telle qu'IBM remette en cause une sphère traditionnelle du pouvoir de l'État, c'est-à-dire les communications, si de simple fabricant de machines, elle devient gestionnaire de réseaux de télécommunication?

Si ce sont les organismes de télécommunications qui lancent et gèrent les satellites, IBM devra dialoguer avec eux. Si leur carence conduit cette compagnie à se substituer à eux, il n'y aura pas de dialogue. Ce serait certes une solution particulièrement efficace mais peu transparente pour les tiers (Nora/Minc, p. 30).

Ainsi l'avènement de la télématique viendrait bouleverser le jeu des acteurs. Devant des constructeurs agressifs dans leur politique de développement, les États seront forcés de défendre leur souveraineté dans le domaine des communications, fief de l'intervention régalienne. L'État français, pour se donner les moyens de se maintenir en position de force, doit fixer les normes d'exploitation et faciliter l'accès aux différents réseaux. Les enjeux se transforment : d'industriels ou commerciaux, ils deviennent culturels. L'empreinte américaine risque de se graver encore plus 
largement dans l'espace culturel français, à travers les banques de données, car avec la télématique et ses réseaux aux multiples ramifications, $c^{\prime}$ est la société entière qui est maintenant concernée.

\subsection{Les technologies d'information, enjeu stratégique pour la modernisation économique et sociale (Rapport Lemoine)}

L'intérêt de ce second rapport procède notamment de son origine, qui coïncide avec un changement notoire sur l'échiquier politique français. En effet, l'année 1981 a été dominée par une élection présidentielle (celle de François Mitterrand), qui devait permettre, pour la première fois depuis le début de la $V^{e}$ République, l'alternance partisane. Plus tard, les élections législatives viendront confirmer la victoire du Parti socialiste qui est le seul à obtenir la majorité absolue des sièges.

L'auteur du rapport, Philippe Lemoine, est de ceux qui ont consacré l'essentiel de leurs réflexions aux enjeux de l'informatisation. II fut notamment l'un des principaux collaborateurs des travaux de la Commission Nora/Minc sur l'informatisation de la société. Au moment de la publication de son rapport, $P$. Lemoine occupait le poste de commissaire du gouvernement auprès de la Commission nationale de l'informatique et des libertés (CNIL).

Engagée en septembre 1982 par le Premier ministre Pierre Mauroy, cette mission d'étude n'avait pas l'ampleur de celle qui avait été confiée à la Commission Nora/Minc. II n'était pas question d'élaborer un projet de société, mais de définir le cadre administratif de la mise en œuvre d'une politique d'utilisation des nouvelles technologies. Ce rapport devait, en outre, permettre au gouvernement de mieux situer son action dans le cadre global défini dans le rapport Nora/Minc.

Le problème étudié dans le rapport Lemoine porte sur I'utilisation effective, en France, des nouvelles technologies. Partant d'une évaluation pragmatique, l'auteur tente d'élaborer une stratégie de modernisation axée sur leur utilisation réelle en vue de relancer "la démarche du progrès". C'est en cela que se manifeste l'originalité des prétentions de ce rapport. II ne s'agit plus de concevoir un projet "abstrait" de société, fondé sur des dispositions aléatoires, mais de définir de façon concrète les adaptations et actions nécessaires à une meilleure gestion des acquis.

Concrètement, le problème constaté à propos de l'utilisation des nouvelles technologies est présenté de la façon suivante : 
En automatisation de la production, les entreprises françaises accumulent un retard inquiétant(...) En informatique, malgré la priorité donnée à ce secteur depuis des années, le degré d'équipement des entreprises et, plus encore, la qualité des applications sont loin d'être satisfaisants. (...) Dans les technologies nouvelles de plus grande diffusion - bureautique, télématique, micro-informatique - la situation paraît plus favorable car le faible coût de ces matériels amène à une expansion rapide des marchés. Mais si l'on regarde les chiffres, on constate un retard de la France encore plus grand qu'en informatique traditionnelle. (...) Plus préoccupant encore : le développement des utilisations "intelligentes" de ces nouvelles technologies paraît s'enliser dans les sables (Lemoine, p. 11-12).

Les conséquences prévisibles d'un tel retard semblent nombreuses. Toutefois, elles sont à nouveau associées principalement au secteur économique. En l'état actuel, caractérisé par un atermoiement à l'égard de l'innovation technologique, il est raisonnable de craindre, pour la France, un enlisement dans la crise. La compétitivité de l'industrie est compromise à cause de son sous-développement en matière d'automatisation, alors que les mesures de correction au niveau de l'emploi ne peuvent bénéficier pleinement de la mobilité issue des nouveaux emplois créés par I'informatisation. On semble néanmoins être également sensible aux conséquences culturelles du retard pris au plan des technologies de l'information. Cela pourrait se manifester par une inadaptation aux modes de raisonnement qu'elles sous-tendent, pourtant nécessaires à la production d'applications originales.

Selon Lemoine, le secteur public, en tant qu'utilisateur privilégié des nouvelles technologies, devrait jouer le rôle d'entraîneur dans la modernisation par l'informatique. Or, sa situation actuelle ne lui permet pas d'exercer adéquatement ce "leadership», déficience qui tiendrait en grande partie aux défauts de la politique nationale en vigueur dans la régie des administrations publiques et à ses conséquences. 
C'est une politique essentiellement négative et réglementaire. (...) C'est ensuite une politique de contrôle au coup par coup des équipements qui nécessite une grande dépense d'énergie, pour des résultats parfois décevants. (...) Cette politique a enfin le défaut d'être centralisatrice et mal adaptée à toutes les nouvelles technologies (Lemoine, p. 30).

Si l'auteur ne remet pas en cause la nécessité de favoriser l'industrie nationale pour éviter la domination d'un constructeur étranger tel qu'IBM, il estime qu'il est grand temps de mettre de côté le problème de l'équipement pour s'attarder davantage à celui de son utilisation. Il devient donc essentiel de faire en sorte que la politique guidant l'exploitation des nouvelles technologies tienne compte des profondes transformations qui affectent le secteur public et de leurs conséquences sur les modes d'utilisation (Lemoine, p. 194).

Le message semble clair. II est nécessaire de modifier la politique nationale d'informatisation de manière à l'adapter aux exigences du mouvement réformiste entrepris par les nouveaux acteurs dans le gouvernement. Guidés par un projet de société précis, les nouveaux décideurs doivent maintenant voir à ce que l'utilisation des technologies de l'information les serve dans les meilleures conditions.

\section{Changement et pouvoir : deux concepts révélateurs}

\subsection{La perception du changement}

À la lecture des deux rapports, le point saillant qui s'impose d'emblée, en ce qui a trait à la conception du changement qui y est préconisée, concerne la forme et l'orientation globale de l'analyse des technologies de l'information par les auteurs. Malgré la filiation reconnue des auteurs et la continuité indéniable de leur problématique, force est de constater la nette démarcation qui les caractérise à cet égard. Ainsi S. Nora et A. Minc mettent l'accent sur les conséquences des nouvelles technologies, notamment la télématique : 
Lorsque les Sumériens inscrivaient les premiers hiéroglyphes sur des tablettes de cire, ils vivaient, sans probablement la percevoir, une mutation décisive de I'humanité : l'apparition de l'écriture. Et pourtant celle-ciallait changer le monde. Aujourd'hui, l'informatique annonce peut-être un phénomène comparable. Les analogies sont frappantes : extension de la mémoire; prolifération et mutation des systèmes d'information; modification éventuelle des modèles d'autorité. La griserie des similitudes peut conduire loin. L'importance d'une telle transformation demeure cependant indéchiffrable pour ceux qui la vivent, sauf à la considérer avec l'oeil de Fabrice à Waterloo (Nora/Minc, p. 116).

De son côté, P. Lemoine insiste plutôt sur les modes d'action à engager aux fins d'une appropriation conséquente des mutations rendues possibles par les technologies d'information :

D'une manière générale, ces différents exemples montrent les bénéfices que I'on peut attendre d'une politique d'utilisation menée "les yeux ouverts" et non "les yeux fermés". "I y a plus à gagner à ce que les différents partenaires prennent conscience à l'avance des impératifs de mutation et de productivité et examinent les moyens d'y faire face, plutot que d'être acculés au changement au moment où plus rien n'est aménageable (Lemoine, p. 25).

L'orientation différente adoptée pour le traitement de leur problématique révèle deux conceptions opposées du changement. S. Nora et A. Minc semblent concevoir le changement comme s'imposant à des acteurs relativement passifs devant l'informatisation de la société. Les nombreuses conséquences de l'informatisation devraient surtout modifier profondément l'articulation de la dynamique sociale (Nora/Minc, p. 114). 
Les effets sociaux de la télématique se situent donc au centre de leur analyse. On y prédit la transformation de la hiérarchie sociale, la modification des conditions de travail et l'insertion des ouvriers dans l'entreprise. De nombreuses fonctions subiront une déqualification rendant caduques certaines expertises pratiquées précédemment, entre autres, par les professionnels de la santé et de l'éducation qui risquent de voir leur profession banalisée.

En dernier ressort, les incidences culturelles constitueront la pierre angulaire des transformations sociales. Devenues le «moteur de l'histoire", leur appropriation s'avérera la condition indispensable à la prise en main de la destinée de la société française (Nora/Minc, p. 115).

Ainsi, le langage informatisé, supplantant les formes traditionnelles, engendrerait de nouveaux modes d'organisation qui modifieraient du coup la structure de la hiérarchie sociale. Le langage devient un enjeu et un vecteur du modèle culturel nouveau.

On accorde donc une très grande importance aux conséquences des nouvelles technologies sur "l'évolution» de la société française. On est tout près d'un déterminisme simple selon lequel la technique ferait inéluctablement l'histoire. Le passage de la société industrielle à la société d'information s'imposerait comme la voie ultime et inévitable.

Bien entendu, il ne faut pas négliger les quelques réserves exprimées tout au long du rapport à l'égard de ce déterminisme technologique. Les auteurs se posent, par exemple, cette question : "Quel est le moteur principal, l'informatisation ou la société?" (Nora/Minc, p. 49). S'ils soutiennent que les transformations sociales ne découlent pas directement de la machine, ils s'empressent d'affirmer son influence sur «l'évolution des procédures et des organisations" (Nora/Minc, p. 49), laquelle serait la source des transformations sociales.

S. Nora et A. Minc dressent le portrait d'une société dépendante des nouvelles technologies, et surtout de la technologie américaine. Convaincus de l'influence déterminante de la télématique sur l'orientation de la société, ils se préoccupent, en priorité, du rétablissement de la souveraineté nationale en réaction à l'omniprésence d'un constructeur étranger. Rappelons-nous que la télématique est présentée comme un moyen privilégié et nécessaire à la sortie de la crise économique, justifiant les moyens à mettre en œuvre pour faciliter le passage au nouveau type de société qu'elle implique. 
Le rapport signé par $P$. Lemoine reflète une conception tout à fait opposée. Soucieux de définir une stratégie d'utilisation des nouvelles technologies, l'auteur est amené à développer une conception du changement qui préconise une collaboration dynamique entre individus et groupes en ce qui concerne l'utilisation de l'informatique. La question ici n'est plus de savoir comment gérer les effets de l'informatisation de la société, mais plutôt d'établir la stratégie qui conviendrait pour orienter l'exploitation des nouvelles technologies vers les finalités qui leur sont attribuées.

Le projet qui anime ce rapport est de rétablir la démarche du progrès. Moins habituelle, moins prestigieuse peut-être que les classiques approches de la technologie, cette optique de l'utilisation se fonde sur un double impératif de lucidité et de démocratie. Face à une rupture sans précédent, elle invite à repartir du pays réel et à l'associer dans toutes ses composantes, à une stratégie inédite de modernisation ILemoine, p. 9).

Le raisonnement est donc inversé. Par exemple, lorsqu'il s'agit d'étudier la relation de l'informatique à l'emploi, il n'est plus question de s'en tenir strictement aux effets de l'introduction des nouvelles technologies mais de découvrir, par un raisonnement interactionniste, les influences réciproques de l'informatique et de l'emploi (Lemoine, p. 21).

Les effets de l'informatisation sur l'emploi auraient rétroactivement des conséquences même sur son expansion. Ainsi, les craintes que soulève l'introduction des technologies d'information au sujet de l'emploi suscitent des résistances tant chez les employeurs que chez les employés. Cette appréhension se traduirait par un freinage de l'informatisation et de l'automatisation avec toutes ses conséquences sur la modernisation et la compétitivité de la société française. Pour cette raison, la mutation technologique doit être accompagnée, sinon précédée, d'une stratégie favorisant la mobilité, la diversification des services et l'enrichissement des tâches. Les changements issus de l'introduction de l'informatique, loin d'être automatiques et déterminés, vont dépendre de la perception qu'en 
auront les individus et les groupes concernés et de la stratégie qu'ils élaboreront en fonction de leurs objectifs.

Dans son analyse, $P$. Lemoine privilégie un aspect de cette dialectique. Comme d'autres avant lui ont déjà porté suffisamment d'attention aux conséquences de l'introduction des nouvelles technologies, il importe dorénavant de procéder à l'ébauche $d$ 'actions à entreprendre en vue $d$ 'instaurer de nouveaux modes de décision. Cela s'impose si l'État veut exercer une emprise sur l'orientation du changement autorisé par l'utilisation de ces nouvelles technologies (Lemoine, p. 36).

Placée en position quasi seigneuriale dans le rapport Nora/Minc, la technologie est ici ramenée à la dimension plus modeste de servante relativement malléable. Certes, la mutation technologique sous-entend de nouveaux équilibres, mais ceux-ci reposent tout autant sur les changements économiques et sociaux dans des interactions complexes où nul facteur ne semble prédominer. En même temps que l'État cherche à s'adapter aux innovations technologiques, il devient impératif de les moduler en fonction des contingences sociales et économiques, par consensus avec les groupes concernés. L'objectif est d'atteindre un niveau efficace de cohérence entre ces différentes variables.

\subsection{La perception du pouvoir}

Les auteurs des deux rapports ont des préoccupations qui dépassent à certains points de vue l'incidence de l'informatique sur la configuration du pouvoir. Elles touchent la mise en forme d'un aménagement structurel particulier des forces en présence en vue de gérer la mutation qu'entraînerait l'introduction des nouvelles technologies. Leurs positions, constatons-nous, pourraient se résumer en deux mots : "régulation" dans le cas de $\mathrm{S}$. Nora et $\mathrm{A}$. Minc et "concertation» dans le cas de P. Lemoine. Certes, ces deux positions ne sont pas nécessairement contradictoires dans la mesure où l'une n'exclut pas l'autre. Pourtant, elles indiquent un ordre de priorité, lequel n'est pas sans conséquence sur l'orientation de leurs propositions.

Dans le cas du rapport Nora/Minc, il n'est pas sans intérêt de signaler la persistance de la vision déterministe des auteurs à propos des conséquences de l'informatique sur le pouvoir. Logiquement, comme elle entraîne des changements prévisibles que les auteurs s'efforcent de circonscrire, la télématique implique également le réaménagement des conditions d'exercice du pouvoir (Nora/Minc, p. 50). 
La "nouvelle informatique" devrait modifier les rapports entre les divers services administratifs et les relations entre administrations centrales et collectivités locales. Plus spécifiquement, l'informatique aurait pour conséquence, dans la situation prévalant au moment du rapport, notamment dans le secteur public, de favoriser les administrations dominantes au détriment des plus démunies (Nora/Minc, p. 104).

II semble donc nécessaire d'infléchir cette orientation en intervenant sur le «mode hiérarchique traditionnel». On entre ainsi de plain-pied dans cette deuxième dimension de la relation pouvoir/nouvelles technologies portant sur la structuration des rapports de force nécessaire à l'informatisation de la société. On retiendra comme point de départ la définition de la notion de pouvoir sur laquelle s'appuient S. Nora et A. Minc.

La notion de pouvoir est double. Elle s'identifie d'une part à une série de rapports, sans cesse mobiles, par lesquels s'ajustent les champs de compétence, de commandement, de domination, à l'intérieur d'un système donné : il s'agit alors de "micropouvoirs". Elle correspond d'autre part aux modes globaux de régulation de la société : marché, plan, rapports de classe; c'est alors le pouvoir qui est en jeu (Nora/Minc, p. 49).

Or, ils retiendront principalement le deuxième aspect en favorisant la régulation exercée par l'État. On estime, bien entendu, que la structure sociale doit permettre l'expression des aspirations et oppositions des différents groupes, mais c'est le contrôle exercé par l'État qu'il convient d'examiner d'abord. "ll $n^{\prime} y$ a pas de spontanéité sans régulation, pas de régulation sans hiérarchisation. L'autogestion, si elle se veut autosuffisance, restera une contre-société marginale" (Nora/Minc, p. 123). La régulation par le centre se pose donc comme indispensable à la socialisation de l'information. II faut gérer les aspirations des groupes en harmonie avec le projet défini par l'État.

L'adoption d'une telle orientation, selon laquelle on privilégie l'intervention de l'État, s'explique de diverses façons. On peut retenir simplement les convictions politiques des cosignataires du rapport ou, plus subtilement, les pressions qui ont pu s'exercer sur 
eux. Cette orientation pourrait découler, comme nous tendons à le démontrer, de la perspective théorique qu'ils adoptent notamment quant aux notions de changement et de pouvoir. Ces motifs nous paraissent appropriés et devraient, comme plusieurs autres non repris ici, être pris en compte dans une appréciation équitable de leur contribution.

Nous allons examiner plus en détail un seul facteur prépondérant qui éclaire presque tous les autres : la défense de la souveraineté de l'État. Cet objectif coïncide avec les préoccupations sociales, politiques et économiques qui dominent les grands débats à l'époque de la rédaction du rapport. II est en effet nécessaire, pour comprendre le sens des recommandations que font les auteurs, de revenir sur le problème fondamental autour duquel s'articule l'ensemble de leur argumentation.

L'attention portée au problème de la souveraineté de l'État français devant l'emprise exercée, dans le domaine de l'informatique, par un constructeur étranger (IBM) implique une stratégie conséquente, imposée par la nécessité de se protéger de l'ennemi qui menace d'investir l'une des composantes essentielles à l'autonomie de la nation: les communications (Nora/Minc, p. 67).

Les risques ne concernent pas uniquement la souveraineté de l'État: à travers le modèle culturel porté par les sources d'informations et les modes de transmission du savoir, c'est la société tout entière qui est en cause. La position dominante des États-Unis en matière de banques de données leur confère, sur la mémoire collective, une emprise redoutable imposant, de ce fait, les traits du modèle culturel américain (Nora/Minc, p. 72).

Abordée sous cet angle, la question de l'informatisation de la société prend une dimension toute particulière. Non seulement la protection de la souveraineté nationale nécessite l'intervention directe de l'État, mais la dimension internationale qui s'y rattache fait de l'État l'acteur principal. Ici, comme d'ailleurs dans un nombre croissant de secteurs, l'internationalité des grands dossiers implique une intervention directe de l'État dans la définition et la mise en œuvre de transformations structurelles au sein même des différents milieux.

L'application d'une telle politique nécessite l'acquisition, au profit de l'État, de puissantes capacités d'arbitrage. "ll faut donner à l'État un instrument de pouvoir (...)" (Nora/Minc, p. 74). La création d'un ministère des Communications devient donc obligatoire pour assurer la coordination de la politique nationale d'informatisation et garantir en même temps la qualité de l'intervention régalienne de l'État. 
En plaçant la souveraineté nationale au centre de leur stratégie, les auteurs sont amenés à favoriser dans leurs propositions la concentration des moyens au profit de l'État. Une fois le problème de souveraineté résolu, on pourra passer aux aspirations conviviales. Cependant, rien de concret n'est proposé à cet égard. Notre analyse de la conception du changement chez S. Nora et A. Minc montre que cet aspect du problème procédera obligatoirement de l'utilisation même de l'outil informatique.

Là encore, P. Lemoine emprunte une voie tout à fait opposée. La modernisation de la société française par l'utilisation des nouvelles technologies ne pourra se réaliser, selon lui, sans tenir compte des initiatives de la base. Il importe donc de définir une stratégie d'ensemble en concertation avec tous les groupes concernés.

P. Lemoine désapprouve la politique menée jusqu'alors dans le secteur public, politique trop axée sur les procédures et compromise par l'imprécision de ses objectifs. Il en résulte un cloisonnement entre les diverses organisations préoccupées uniquement des intérêts de leur milieu et incapables de coordonner leurs actions avec celles de leurs partenaires. Cette situation se traduit par des rapports conflictuels entre les organisations, jalouses de leurs prérogatives au détriment d'une exploitation efficace du potentiel informatique.

À l'opposé de cette politique, l'auteur en développe une autre fondée essentiellement sur la concertation. Pour «mobiliser les vouloirs", nécessaires à un développement réel du tissu informatique, il faut substituer au contrôle réglementaire, privilégié jusqu'alors, une implication des divers groupes dans la définition et la réalisation d'une politique informatique globale (Lemoine, p. 83).

La consultation des utilisateurs permettrait de mettre au point des méthodes d'exploitation de l'outil qui seraient plus adéquates et mieux intégrées aux réalités concrètes des opérations. En suscitant la concertation entre les organisations patronales, syndicales et les groupements d'utilisateurs, il devient possible de pallier le cloisonnement qui, en raison des luttes stériles qu'il entraîne, paralyse actuellement l'utilisation des nouvelles technologies. On arriverait ainsi à une stratégie d'ensemble qui tiendrait compte notamment de la transformation du travail et du problème de l'emploi. Bref, il s'agit de suppléer au fractionnement généré par la politique menée jusqu'à maintenant en développant des modes de coopération pour la mise en œuvre de projets harmonisés. Cela doit se traduire par la mise en œuvre d'une démarche contractuelle, la clarification des axes politiques et la 
participation des différents utilisateurs à la préparation de ce cadre d'ensemble.

Les stipulations des contrats d'achat de matériel devant couvrir non seulement les conditions conformes à une politique industrielle donnée, mais spécifier également avec précision I'utilisation des appareils, ceci rend nécessaire de disposer d'une instance compétente pour classer les priorités, définir des objectifs et arbitrer les conflits (Lemoine, p. 83).

Cette instance est le Commissariat aux technologies d'information. Sa structure et sa mission l'apparenteraient au Commissariat général du plan. Institution-phare, elle fonctionnerait comme un guide pour l'emploi des nouvelles technologies, sous l'impulsion d'un consensus d'orientation regroupant les différents partenaires autour d'une stratégie commune.

Ainsi, contrairement à S. Nora et A. Minc, P. Lemoine s'est attardé à ce que les premiers appelaient les "micro-pouvoirs", c'est-à-dire à cet aspect du politique dans lequel l'activité des différents groupes influence considérablement les résultats des politiques menées par l'État. En ce domaine, la régulation dépasse la volonté d'un nombre restreint d'acteurs centraux et repose davantage sur l'engagement et la collaboration d'une multitude de groupes aux intérêts variés et parfois contradictoires. L'intervention de l'État se justifie davantage, ici, dans les actions et les orientations adoptées en son sein en faveur du développement de l'informatique sans recourir au contrôle et à la réglementation de l'activité des autres intervenants.

\section{Deux visions, deux projets}

Ayant pour mission de dégager les lignes directrices de la politique française relative à l'emploi des technologies nouvelles, les auteurs des deux rapports adoptent des orientations opposées. Alors que $\mathrm{S}$. Nora et $\mathrm{A}$. Minc semblent postuler un déterminisme technologique, $P$. Lemoine affirme, au contraire, le caractère prépondérant de la stratégie d'utilisation déployée par les divers acteurs concernés.

Les conséquences inhérentes aux positions divergentes prises par les auteurs des deux rapports sont éloquentes. Elles concernent, entre autres, la nature des propositions contenues dans ces rapports, et l'orientation des actions suggérées. Afin d'illustrer de manière significative les effets pratiques de chacune des deux orientations, nous examinerons le rôle dévolu à l'État dans la mise en œuvre de la politique préconisée et, 
conséquemment, l'orientation privilégiée en matière de centralisation/décentralisation. En fait, nous explorerons de cette manière la relation pouvant s'établir entre l'informatique et la démocratie ${ }^{21}$.

Les mesures concrètes proposées dans le rapport Nora/Minc se définissent en fonction de deux scénarios: celui du "Tout-État" et celui de la "décentralisation extrême». La position retenue par les auteurs se situerait quelque part à mi-chemin entre ces deux extrêmes.

Si les pouvoirs publics laissent l'informatique pénétrer en désordre, ils figent l'avenir. $\dot{A}$ l'inverse, aucun scénario global ne peut être imposé à partir d'un centre unique, sans étouffer la société ou bloquer l'État. II faut donc concilier un maximum de liberté et un minimum de coordination, faciliter le changement plutôt que l'imposer (Nora/Minc, p. 101).

Les auteurs optent ainsi pour l'établissement d'un compromis entre les préoccupations régaliennes de l'État et la liberté conviviale de la société. Ils diront notamment, à propos de l'industrie de la mini et la péri-informatique, que "l'État doit mener deux politiques, l'une d'incitation, l'autre de précaution" (Nora/Minc, p. 94). Pourtant, obnubilés par la portée des effets de la télématique, les auteurs semblent davantage préoccupés par la mise en œuvre d'instruments favorables à la réglementation de l'industrie par l'État, de sorte qu'on arrive à se demander ce qu'il advient de leurs aspirations conviviales.

En conférant à l'État, notamment par l'intermédiaire d'un ministère des Communications, le contrôle sur les banques de données et sur la définition des normes d'utilisation du réseau, ne jette-t-on pas les bases d'une centralisation poussée, susceptible d'encadrer finalement la société entière? D'ailleurs, cette centralisation n'est-elle pas, à son tour, considérée comme s'imposant d'elle-même? (Nora/Minc, p. 106)

21. Voir à ce sujet le texte de M. Finlay, «Développement social et nouvelles technologies de l'information : approche discursive», dans K. Fall et G. Vignaux (sous la direction de), L'informatique en perspective, Sillery, Presses de l'université du Québec, 1989. 
La position centrale accordée, dans leur analyse, à la télématique est un indicateur manifeste de la prédilection des auteurs pour la centralisation. L'interconnectabilité qu'elle suppose demande la mise en place d'une structure centrale de transmission des données. Elle implique surtout la gestion, au niveau national, d'un réseau permettant d'imposer des normes de transmission et d'utilisation qui serviraient à renforcer le pouvoir de l'administration centrale.

Cette propension à la centralisation se révèle de manière encore plus marquée lorsqu'on constate le faible intérêt que portent les auteurs à la micro-informatique, souvent perçue comme une avenue favorable à la décentralisation. En se préoccupant davantage de la souveraineté de l'État dans le cadre d'une application plutôt centralisatrice (la télématique) et en négligeant d'explorer une technique favorisant l'autonomie (la microinformatique), les auteurs paraissent définitivement plus portés vers les mérites de la centralisation.

De son côté, $P$. Lemoine critique sévèrement l'orientation donnée à la politique technologique. Ainsi, il remettra en cause le fractionnement et le cloisonnement des organismes compétents en matière d'utilisation des technologies d'information. Mais il adresse ses reproches les plus sévères aux finalités de leurs actions.

Dans leur équilibre actuel, les
organismes de la politique d'utilisation
se définissent en effet par le jeu de trois
verbes : interdire ou réglementer; faire
ou expérimenter; faire-faire. Sur ces
trois verbes se sont greffées deux
préoccupations complémentaires :
savoir-faire (recherche) et faire-savoir
(information et sensibilisation) (Lemoine,
p. 54).

Cette optique ne répondrait plus aux besoins de l'informatisation, parce que les questions qu'elle pose nécessitent de nouvelles approches. II importe maintenant de réduire les incertitudes qu'elle comporte par la définition d'une stratégie d'ensemble. "Aujourd'hui, la question principale n'est pas de réglementer, d'expérimenter ou de faire-faire. Le véritable impératif c'est de dégager des vouloirs" (Lemoine, p. 56). 
À terme, c'est à la décentralisation de la vie économique, administrative et sociale qu'il faut aspirer. Elle seule permettra une réelle prise en charge collective et universelle des grandes mutations rendues possibles par le développement des nouvelles technologies. II importe de faire obstacle à la globalisation des problèmes inhérente à la gestion centralisée de l'informatisation. $\dot{A}$ cette fin, il faut encourager les initiatives au niveau local, favoriser l'équipement des collectivités locales et procéder au transfert réel de compétences de l'État vers ces collectivités locales ${ }^{22}$.

Seule la coopération entre les divers intervenants permettrait de pallier les embûches que rencontre l'éclosion d'une société informatisée. Or, cette coopération ne peut être imposée d'en haut; elle repose sur la concertation entre des groupes autonomes, conscients de leurs besoins, responsables de leur gestion et sensibles à leur dépendance réciproque. Au lieu de chercher à casser, par une réglementation tatillonne, la "dynamique motrice» qui anime chacun des secteurs, on doit encourager l'expression de celle-ci en vue de dégager les grands axes d'une politique $d^{\prime} u t i l i s a t i o n$ des nouvelles technologies ${ }^{23}$. Ici, le centre ne doit plus s'imposer, il doit être un lieu d'échange, de concertation permettant de définir les priorités et d'établir les objectifs qui doivent mener à une utilisation fonctionnelle de l'informatique. Toutes ces mesures présentées comme essentielles à la modernisation de la société française expriment une volonté réelle de prendre en main la mutation technologique.

\section{Conclusion}

Notre analyse tend à démontrer comment l'écart entre les deux rapports, au plan de la stratégie, résulte d'une perception différente des phénomènes du changement et du pouvoir. II en ressort que les plus lourdes conséquences de l'informatisation de

22. Rappelons que le dépót du rapport Lemoine a coïncidé avec l'amorce de la réforme de décentralisation menée par le gouvernement socialiste. De plus, les socialistes avaient annoncé leur volonté de démocratiser l'informatique, de ne plus imposer mais de proposer. J.P. Chevènement, ministre de la Recherche et de la Technologie, Le Monde, 23 septembre 1981, p. 1.

23. I. A. Litvak a démontré, pour le Canada, comment une politique de développement technologique peut être couteuse pour les entreprises bénéficiaires. I. A. Litvak, "Public policy and high technology SMES : The government embrace", Administration publique du Canada, vol. 35, n 1, 1992, p. 22-38. 
la société ne procèdent pas de l'introduction de l'outil en lui-même, mais des projets de société qu'il engendre et de la conception de la réalité qui sous-tend ces projets. II semble en effet, si notre analyse est juste, que la conception objectiviste transmise par Nora et Minc dans leur étude les porte à favoriser une plus grande centralisation, un contrôle plus étroit sur la société informatisée. À l'opposé, la conception subjectiviste que Lemoine préconise le conduit à privilégier la décentralisation et la concertation ${ }^{24}$. Or, I'histoire récente nous enseigne que si le rapport Nora/Minc a présidé longtemps au processus d'informatisation de la société française, les orientations prônées par Lemoine reçoivent une attention de plus en plus favorable ${ }^{25}$.

L'attrait du rapport de Lemoine vient précisément de ce qu'en réhabilitant la volonté humaine, il se démarque du déterminisme technologique qui semble caractériser le rapport Nora/Minc. Pour l'auteur, ce sont moins les transformations de la société par l'informatique que les transformations de l'informatique par la société ${ }^{26}$ qui doivent nous préoccuper. Les formes de mutation issue des développements dans les technologies de l'information dépendent de l'usage qu'en font les individus et les groupes et selon lequel ils orienteront leur action ${ }^{27}$. Dans cette optique, le changement est considéré comme issu des intentions des acteurs et n'est plus perçu comme s'imposant à la volonté des individus. "La force de l'impact tiendrait non pas à la technique

24. Voir à ce sujet l'article de C. Sitarski, "Conceptual issues in the study of centralization and information technologys, Administration publique du Canada, vol. 34, n 4, 1991, p. 641-663.

25. Voir notamment M. Crozier, Etat modeste, État moderne. Stratégie pour un autre changement, Paris, Fayard, 1987.

26. A. Corten, M. B. Tahon (sous la direction de ), La radicalité du quotidien : Communauté et informatique, Montréal, VLB Éditeur, 1987.

27. Voir à ce sujet l'article de J. Mercier où il démontre, en rapport à l'informatisation de l'administration publique québécoise, que l'autonomie des individus face à l'outil informatique est plus déterminante que l'outil lui-même : J. Mercier, «Répercussions de l'informatisation dans le secteur public québécois", Politique, $n^{\circ} 18,1990$, p. 9-32. 
comme telle, mais aux stratégies d'usage, de pouvoir, de résistance des utilisateurs de ces techniques ${ }^{28}$ ".

Dans ces conditions, c'est à l'éclatement du pouvoir qu'il convient de s'intéresser. La mission de l'État n'est plus d'arbitrer les rapports de force de manière autoritaire par une réglementation mécanique où l'ordinateur est d'ailleurs perçu comme un puissant outil de contrôle. En reconnaissant l'éclatement du pouvoir, l'État devrait s'employer à définir des lieux de concertation où, par un processus d'échange, de négociation et de marchandage, se détermineraient les contours de la société informatisée.

Trop souvent magnifiée, l'informatique retrouve une dimension plus réaliste chez Lemoine qui ne la considère pas comme au-delà de la dynamique sociale, son potentiel ne pouvant se réaliser que si son introduction est accompagnée d'une transformation des rapports sociaux et politiques ${ }^{29}$.

Bref, il est possible d'exploiter l'argument informatique pour justifier le recours à la centralisation et à la réglementation autoritaire. Cependant, aucun déterminisme n'y mène et il peut être tout aussi justifié de favoriser la concertation et la décentralisation. L'informatisation de nos sociétés peut représenter une occasion nouvelle de redéfinir nos règles du jeu, de reconsidérer l'état de nos rapports sociaux. Tout cela repose en fait sur notre conception de ce qu'est la réalité et de ce qu'elle devrait être.

28. A. Billette, M. Cantin, Stratégie informatique et son impact sur l'organisation du travail dans l'assurance generale, Rapport de recherche subventionné par Travail-Canada, Département de sociologie, université Laval, avril 1986, p. 21, cité par : G. Laflamme, G. Vallée, Nouveaux modes régulateurs et changements technologiques : cas et problématique, Rapport de recherche subventionné par le Conseil québécois de la recherche sociale, Département des Relations industrielles, université Laval, 30 septembre 1987.

29. Voir P. Breton, S. Proulx, L'explosion de la communication : la naissance d'une nouvelle idéologie, Paris, Montréal, La Découverte, Boréal, 1989. 